\title{
EFFECTS OF GRAVITY ON BUBBLE FORMATION IN AN ANNULAR JET
}

\author{
R.A. Koepp, R.N. Parthasarathy and S.R. Gollahalli \\ School of Aerospace and Mechanical Engineering \\ The University of Oklahoma \\ Norman, OK 73019 \\ E-mail: rparthasarathy@ou.edu; Phone: 405-325-1735
}

The effects of gravity on the bubble formation in an annular jet were studied. The experiments were conducted in the 2.2-second drop tower at the NASA Glenn Research Center. Terrestrial gravity experiments were conducted at the Fluid Dynamics Research Laboratory at the University of Oklahoma. Stainless steel tubing with inner diameters of 1/8" (gas inner annulus) and 5/16" (liquid outer annulus) served as the injector. A rectangular test section, 6" x 6" x 14" tall, made out of half-inch thick Lexan was used. Images of the annular jet were acquired using a high-speed camera. The effects of gravity and varying liquid and gas flow rates on bubble size, wavelength, and breakup length were documented.

In general, the bubble diameter was found to be larger in terrestrial gravity than in microgravity for varying Weber numbers $(0.05-0.16$ and $5-11)$ and liquid flow rates $(1.5 \mathrm{ft} / \mathrm{s}-3.0 \mathrm{ft} / \mathrm{s})$. The wavelength was found to be larger in terrestrial gravity than in microgravity, but remained constant for varying Weber numbers. For low Weber numbers $(0.05-0.16)$, the breakup length in microgravity was significantly higher than in terrestrial gravity. Comparison with linear stability analysis showed estimated bubble sizes within $9 \%$ of experimental bubble sizes. Bubble size compared to other terrestrial gravity experiments with same flow conditions showed distinct differences in bubble size, which displayed the importance of injector geometry on bubble formation. 


\section{Effects of Gravity on Bubble Formation in an Annular Jet}

R. A. Koepp, R. N. Parthasarathy, and S. R. Gollahalli

School of Aerospace and Mechanical Engineering

University of Oklahoma

Norman, OK

Acknowledgments:

John McQuillen, Brian Motil

NASA GRC 


\section{OBJECTIVES}

- To study the effects of gravity on periodic bubble formation in an annular jet.

- To measure bubble size, frequency, and breakup length in identical set-ups in microgravity and terrestrial conditions. 


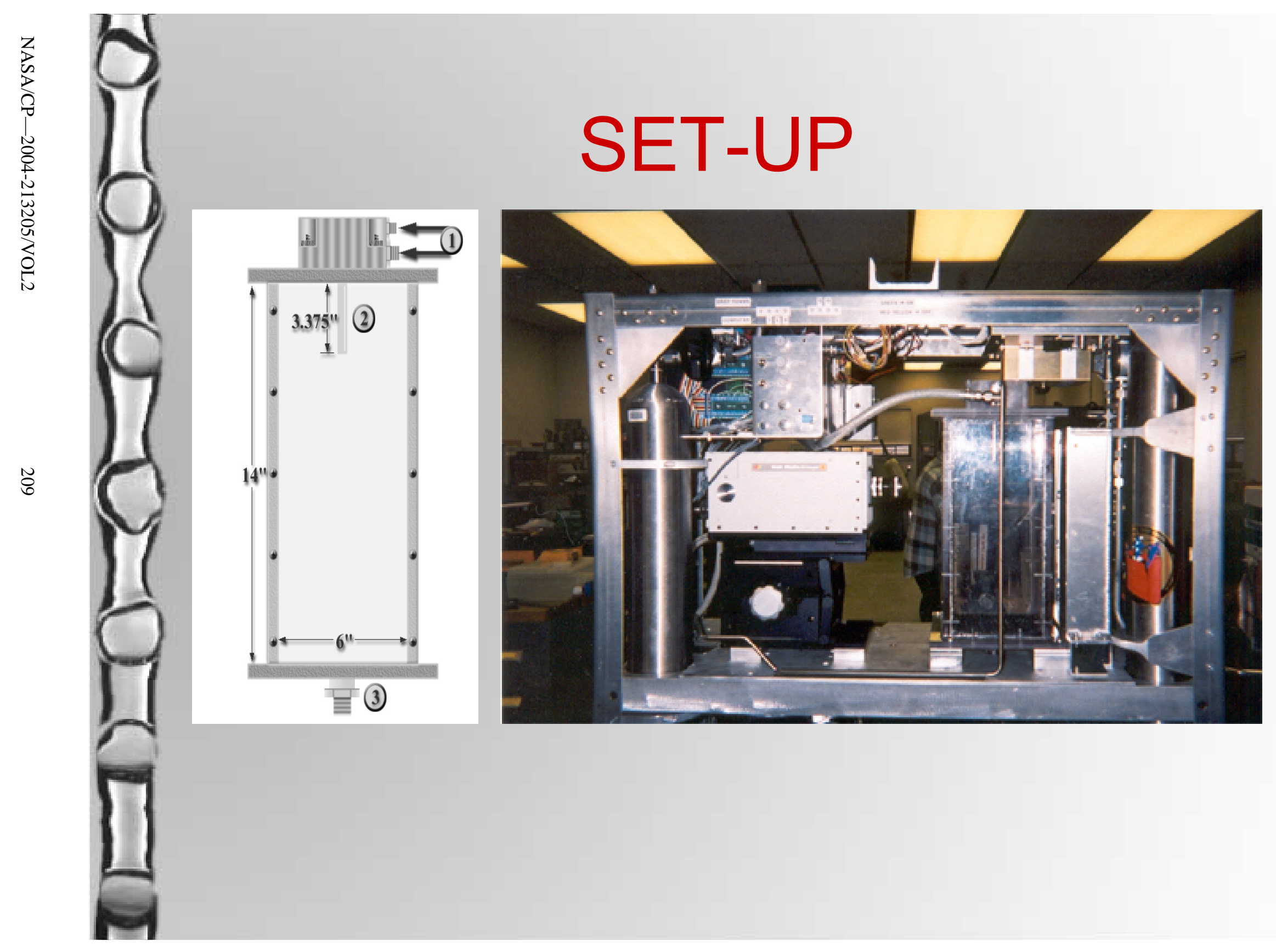




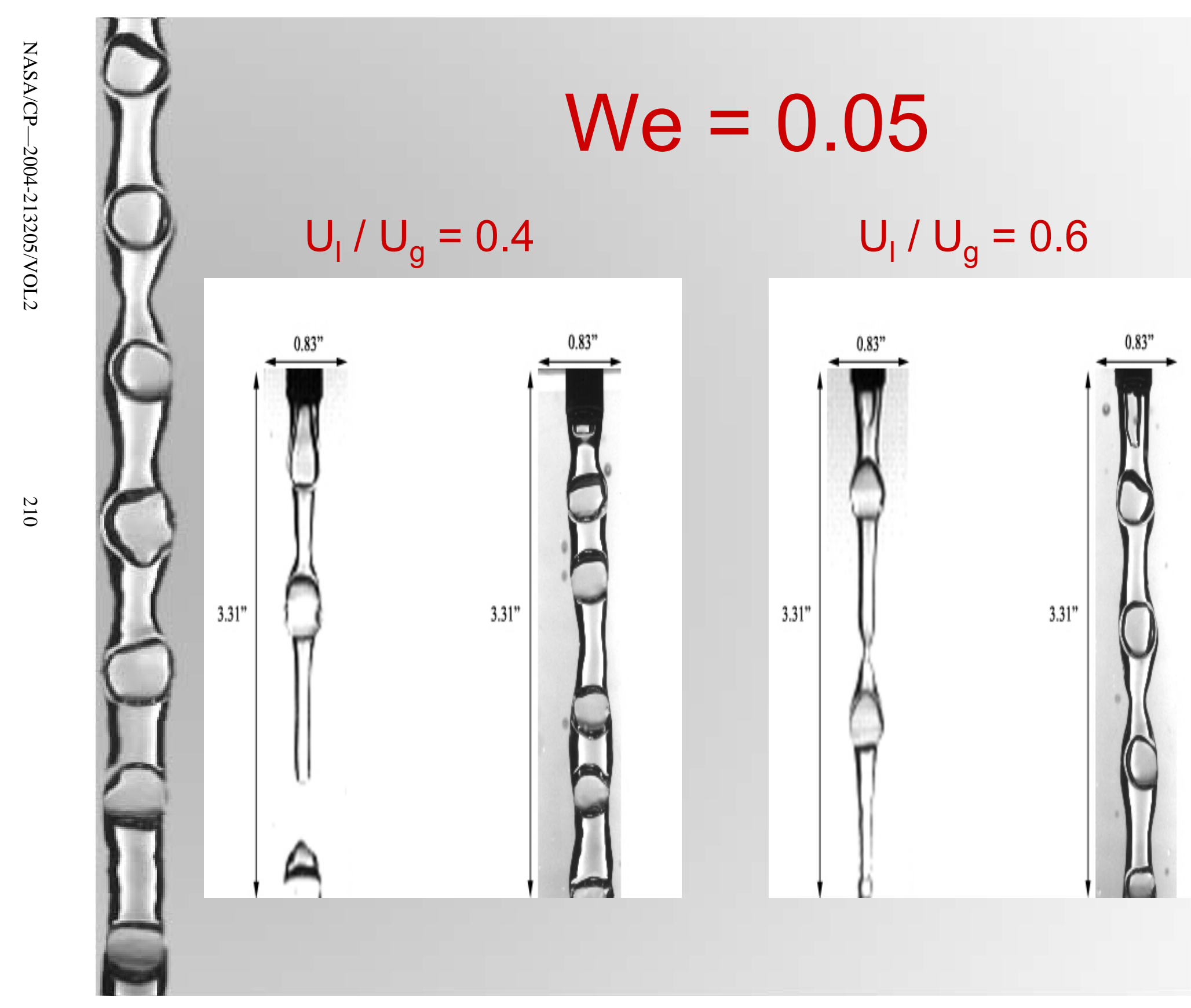




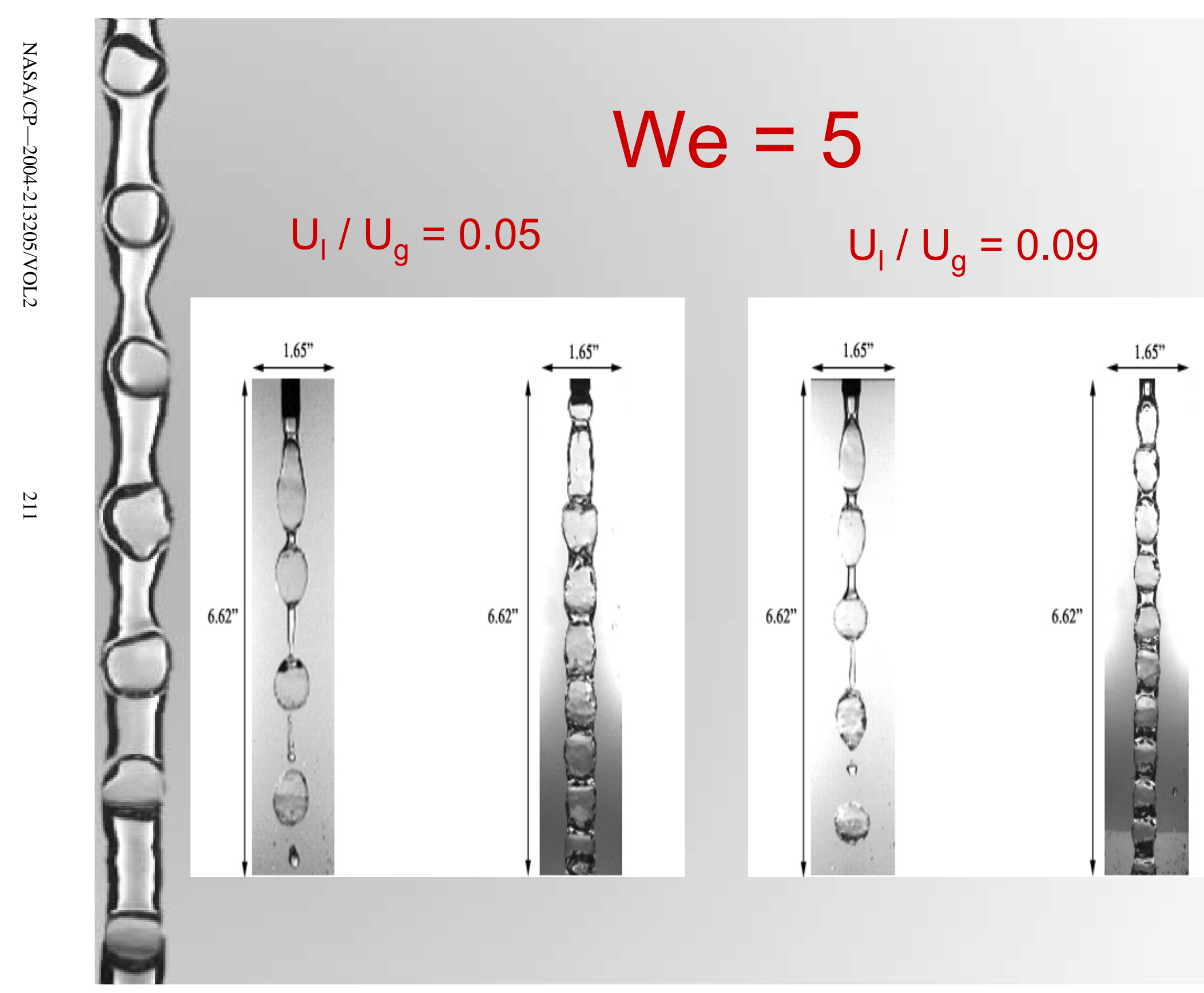




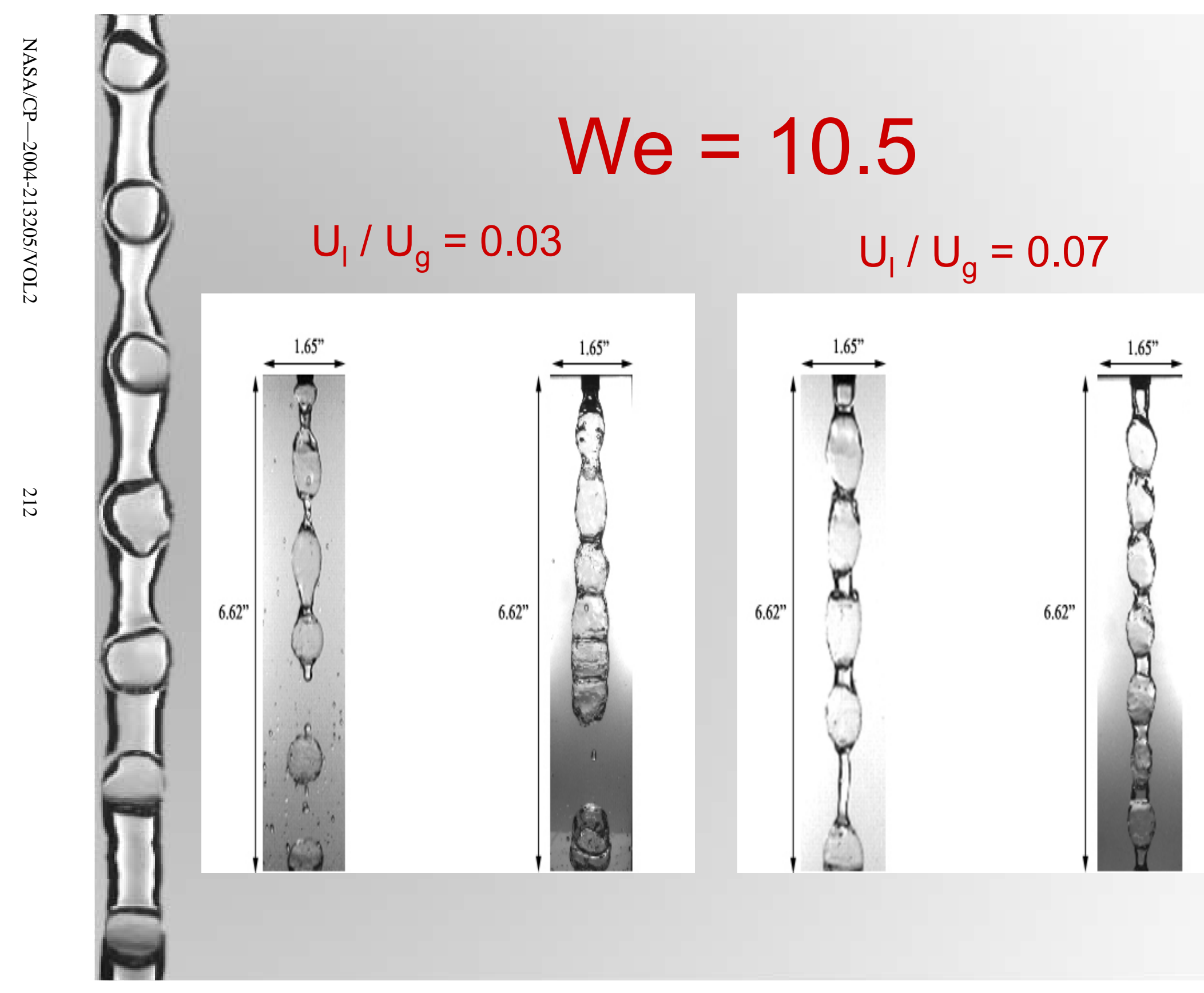




\section{Normalized Bubble Size}

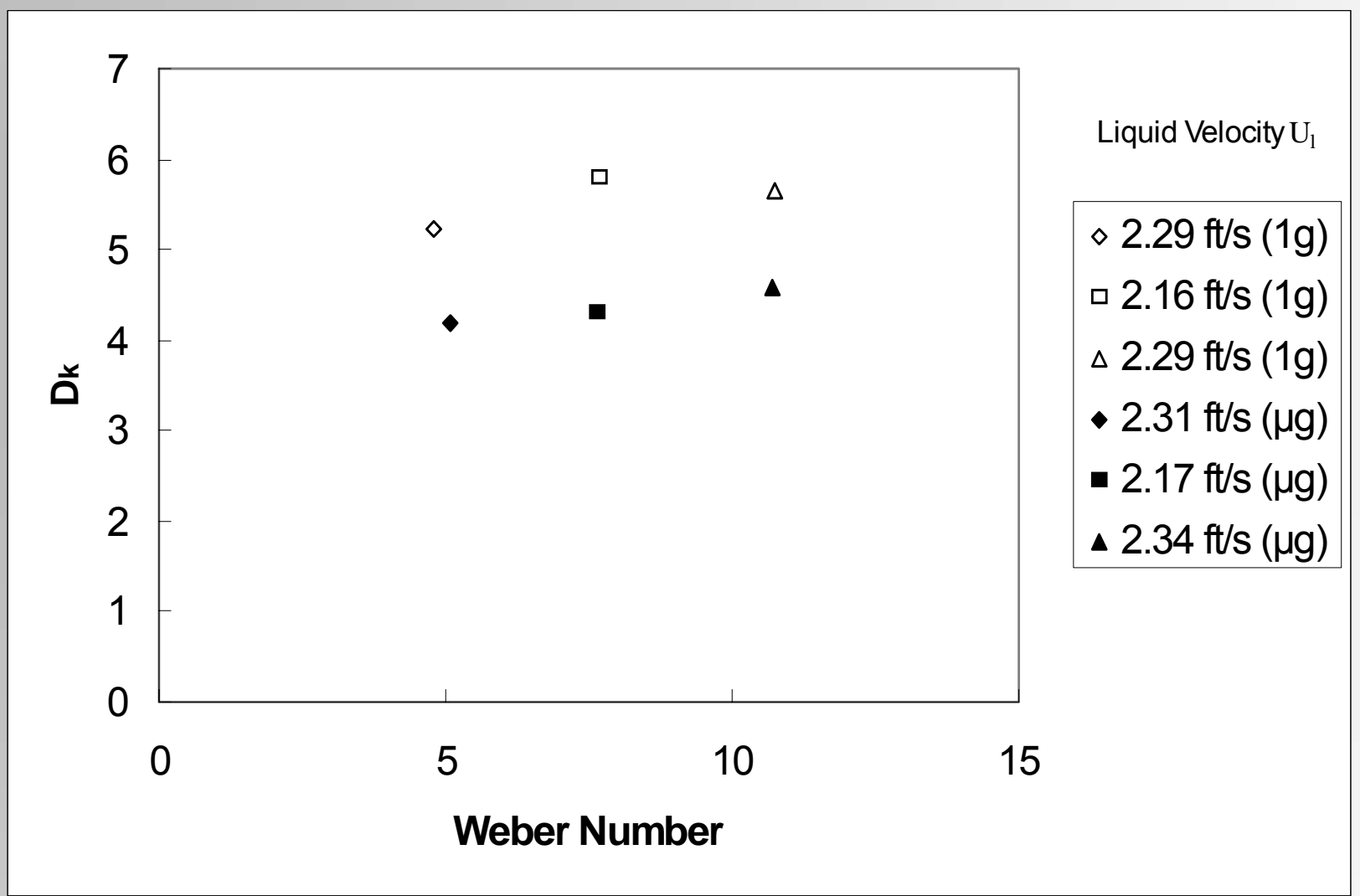




\section{Normalized Breakup Length}

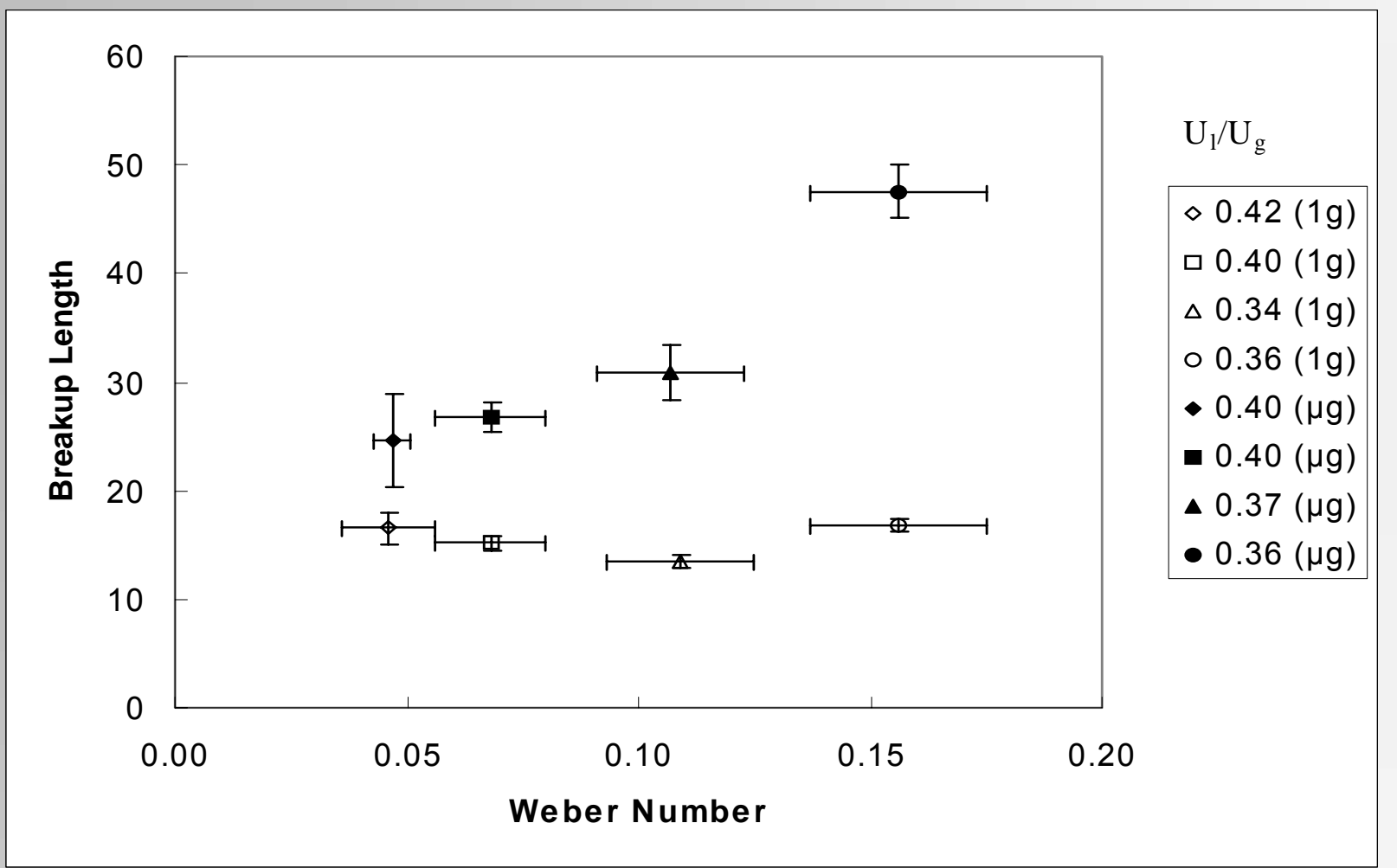




\section{Conclusions}

Bubble size was larger in normal gravity than in microgravity due to reduced relative velocities and buoyancy effects.

Wavelength was larger in normal gravity due to the stretching of the jet.

Acceleration due to gravity caused the jet to thin and pinch off leading to shorter breakup lengths in normal gravity.

Breakup was not observed in microgravity at large Weber numbers. 